\title{
An Introduction to Mössbauer Spectroscopy
}




\section{Contributors}

\section{Jacques Danon}

Professor, Centro Brasileiro de Pesquisas Fisicas, Rio de Janeiro, Brasil

\section{Peter G. Debrunner}

Associate Professor of Physics, Department of Physics, University of Illinois, Urbana, Illinois 61801

\section{Hans Frauenfelder}

Professor of Physics, Department of Physics, University of Illinois, Urbana, Illinois 61801

\section{Ulrich Gonser}

Professor, Institut für Metallphysik und Metallkunde, Universität des Saarlandes, 66 Saarbrücken 15, Germany

\section{David W. Hafemeister}

Associate Professor of Physics, Department of Physics, California State Polytechnic College, San Luis Obispo, California 93401

\section{Rolfe H. Herber}

Professor of Chemistry, School of Chemistry, Rutgers University, The State University of New Jersey, New Brunswick, New Jersey 08903

Robert L. Ingalls

Associate Professor of Physics, Department of Physics, University of Washington, Seattle, Washington 98105

\section{Leopold May}

Associate Professor of Chemistry, Department of Chemistry, The Catholic University of America, Washington, D.C. 20017

\section{Jon J. Spijkerman}

Research Physicist, Analytical Chemistry Division, National Bureau of Standards, Washington, D.C. 20234

John C. Travis

Research Physicist, Analytical Chemistry Division, National Bureau of Standards, Washington, D.C. 20234 


\title{
An Introduction to Mössbauer Spectroscopy
}

\author{
Edited by \\ Leopold May \\ Department of Chemistry \\ The Catholic University of America \\ Washington, D.C.
}


Library of Congress Catalog Card Number 76-137011

ISBN 978-1-4684-8913-2 ISBN 978-1-4684-8911-8 (eBook)

DOI 10.1007/978-1-4684-8911-8

(C) 1971 Plenum Press, New York

Softcover reprint of the hardcover 1st edition 1971

A Division of Plenum Publishing Corporation

227 West 17th Street, New York, N.Y. 10011

United Kingdom edition published by Plenum Press, London

A Division of Plenum Publishing Company, Ltd.

Donington House, 30 Norfolk Street, London W.C. 2, England

All rights reserved

No part of this publication may be reproduced in any form without written permission from the publisher 


\section{Preface}

The initial impetus for this text occurred when we were searching for a single book that could be recommended to the attendees at the Mössbauer Spectroscopy Institute at The Catholic University of America. This Institute is an introductory course on the theory and interpretation of Mössbauer spectroscopy for workers in industrial, academic, and government laboratories. None of the books available adequately covered the breadth and scope of the lectures in the Institute. A list of these books and review articles is included in Appendix C. To meet our needs, we undertook the creation of this text.

The chapters are based upon the lectures given at the various Institutes from 1967 to 1969. Most of the lectures were recorded and transcripts sent to the lecturers, who then prepared the manuscripts, using the transcripts as a guide so as to retain the style developed during the lecture. Each chapter is written in the style of the authors. As the editor, my main task was to maintain uniformity of format and nomenclature. A list of nomenclature used in this volume is reproduced in Appendix A. We hope that this list will be used particularly by new investigators and teachers of Mössbauer spectroscopy so that future literature will employ a uniform system.

The text is written primarily to introduce scientists, both at the graduate student level and in active research, to areas in which Mössbauer spectroscopy may be of assistance. Each author is an active research worker, and many are regarded as leaders in their particular aspect of the subject matter. They bring to their writing both specific knowledge of their research and the desire to transmit adequately the results and principles to the reader.

I wish to thank the authors for their efforts and suggestions in making this endeavor possible and also the secretarial staff at the Chemistry Department of Catholic University for their assistance, particularly Mrs. Olivia Messer and Miss Judy Williams. 


\section{Contents}

\section{Chapter 1}

Introduction to the Mössbauer Effect................................. 1

by Peter G. Debrunner and Hans Frauenfelder

1. Pictorial Description ......................................... 1

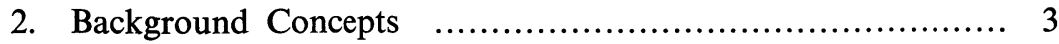

2. 1. Natural Line Width ................................... 3

2. 2. Recoil Energy Loss....................................... 5

2. 3. Resonance and Resonance Fluorescence ............... 6

2.4. Doppler Broadening ...................................... 9

2. 5. Einstein Solids ........................................ 10

2.6. Recoil-Free Emission of Gamma Rays ................. 12

3. The Mössbauer Effect ............................................. 14

3. 1. Pictorial Approach ........................................ 14

3. 2. Observation of the Mössbauer Effect.................... 15

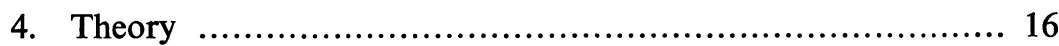

\section{Chapter 2}

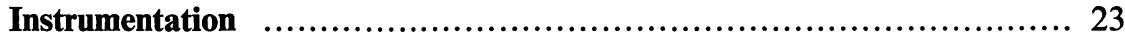

by Jon J. Spijkerman

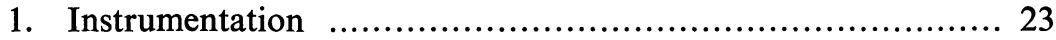

1. 1. Gamma-Ray Detection ............................... 24

1.2. Doppler Velocity Drive ................................ 26

2. Accuracy and Precision ...................................... 28

2. 1. Calibration Methods ...................................... 29

2.2. Time of Counting ....................................... 30

3. Experimental Techniques ..................................... 30

3. 1. Sources .................................................. 30

3. 2. Mössbauer Absorbers ..................................... 32

3. 3. $f$-Factor Measurements ................................. 33

3.4. Variable Temperature ....................................... 34

3. 5. Scattering Methods ....................................... 38

4. Applications of Computers to Mössbauer Spectra ............. 41

4. 1. Computation of Mössbauer Spectra from a Theoretical Model 
4. 2. Curve Fitting of Mössbauer Data by Least-Square Analysis ............................................... 42

4. 3. Curve Fitting of Mössbauer Data by Constrained Least-Square Analysis................................. 43

Chapter 3

Nuclear Properties Determined from Mössbauer Measurements ........ 45 by David W. Hafemeister

1. A Phenomenological View of the Hyperfine Interactions..... 46

2. Differences in the Nuclear Charge Radius $\Delta R / R \ldots \ldots \ldots \ldots . . .51$

2. 1. $\Delta|\psi(0)|^{2}$ from "Pure Ionic" Valence States .............. 51

2. 2. $\Delta|\psi(0)|^{2}$ from Band Theory ............................... 52

2. 3. $\Delta|\psi(0)|^{2}$ Caused by Overlap ............................ 52

2.4. $\Delta|\psi(0)|^{2}$ from the Shielding of $p$ Holes .................. 53

2. 5. $\Delta R$ Ratios Method ......................................... 54

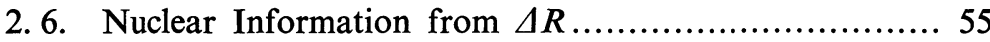

3. Nuclear Quadrupole Moments $Q$ and Spins $I$.............. 56

3. 1. Quadrupole Moment Ratios $Q^{*} / Q \ldots \ldots \ldots \ldots \ldots \ldots \ldots \ldots . \ldots . \ldots . \ldots 7$

3. 2. Direct Determination of Quadrupole Moment Values................................................. 60

3. 3. Direct Determination of Nuclear Spins .................. 62

4. Nuclear Magnetic Dipole Moments ........................... 62

5. Nuclear Lifetimes .................................................. 65

6. Internal Conversion ............................................. 67

7. Parity, Multipole Mixing, and Time Reversal.................. 68

7. 1. Mössbauer Observation of Parity Nonconservation ... 68

7. 2. Multipole Mixing and Time Reversal ................. 70

8. Nuclear Reactions and Devices ................................ 71

\section{Chapter 4}

The Electric Field Gradient Tensor …............................... 75

by John C. Travis

1. The "Standard Form" EFG Tensor Due to a Single-Point Charge ................................................... 75

2. The Ligand Contribution ..................................... 78

3. The Valence Electron Contribution $\ldots \ldots \ldots \ldots \ldots \ldots \ldots \ldots \ldots \ldots . \ldots \ldots$

4. Quadrupole Splittings........................................... 87

4. 1. The Nuclear Quadrupole Interaction .................. 88

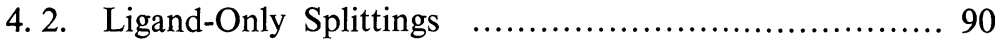

4. 3. Valence-Only Splittings …............................ 93

4. 4. Ligand-Valence Combined Splittings ..................... 95

5. Potpourri ....................................................... 96 
5. 1. Compounds with Internal Magnetic Fields ............. 97

5. 2. Applied Magnetic Fields .................................. 97

5. 3. The Gol'danskii-Karyagin Effect ......................... 98

5.4. Single Crystal Samples ................................... 98

6. The Utility of EFG Information ................................ 99

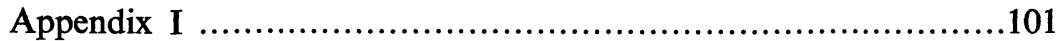

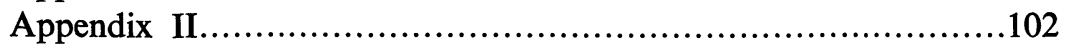

Chapter 5

Application to Solid-State Physics .....................................104

by Robert L. Ingalls

1. Isomer Shift ........................................................104

2. Magnetic Hyperfine Structure ....................................109

3. Electric Quadrupole Interaction ..................................114

4. Lattice Dynamics .................................................118

Chapter 6

Application to Coordination Chemistry …................................120

by J. Danon

1. Crystalline Structure ............................................124

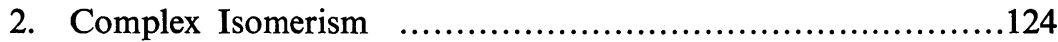

2.1. Cis-Trans Isomerism ...................................125

2. 2. Ligand Linkage Isomerism ..............................126

2. 3. Spin-State Equilibria ..................................127

3. Structure of Complicated Complex Compounds .................128

4. Electronic Structure of Molecules ...............................131

4. 1. Using Ligand Field Theory ..............................131

4. 2. Using Molecular Orbital Theory ..........................133

4. 3. Using the Spin Hamiltonian.................................134

\section{Chapter 7}

Application to Organometallic Compounds …...........................138

by R. H. Herber

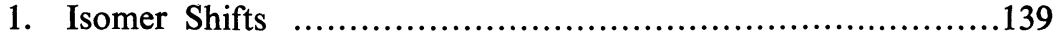

1.1. Organoiron Compounds....................................139

1.2. Organotin Compounds ....................................140

2. Quadrupole Splitting ...........................................141

2. 1. Organoiron Compounds..................................141

2. 2. Organotin Compounds ....................................143

3. Conformational Studies ........................................147

3. 1. Cyclooctatetraene Iron Tricarbonyl $\left[\mathrm{COTFe}(\mathrm{CO})_{3}\right] \ldots . .147$

3. 2. $\left[\pi-\mathrm{C}_{5} \mathrm{H}_{5} \mathrm{Fe}(\mathrm{CO})_{2}\right]_{2} \quad \mathrm{SnCl}_{2}$ and Related Molecules .........150

4. Conclusion ........................................................152 


\section{Chapter 8}

Mössbauer Spectroscopy and Physical Metallurgy

by U. Gonser

1. Mössbauer Application to Physical Metallurgy ................156

1. 1. Precipitation in the $\mathrm{Cu}-\mathrm{Fe}$ System ......................157

1. 2. Phase Transition in Stainless Steel .......................161

1. 3. Internal Oxidation Studies...................................163

1. 4. Magnetic Properties in Au-Fe Alloys ...................163

1. 5. Near-Neighbor Interaction in $\alpha$-Fe-Mo Alloys .......165

1. 6. Near-Neighbor Interaction in Fe-C Alloys ..............166

1. 7. Order-Disorder in $\mathrm{FeAl}$ and $\mathrm{Fe}_{3} \mathrm{Al}$...................170

1. 8. Thin Films and Superparamagnetism ...................171

1.9. Mössbauer Effect as a Nondestructive Analytical Tool

2. Physical Metallurgy Considerations Concerning the Mössbauer Effect ........................................173

3. Summary ......................................................176

Chapter 9

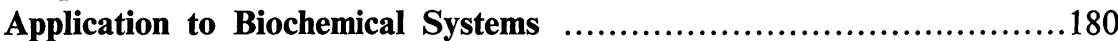

by Leopold May

1. Hemoproteins ................................................182

2. Experimental Conditions .....................................183

2. 1. Enrichment ..........................................183

2. 2. Effect of Thickness .....................................184

2. 3. State of the Absorber ...................................185

3. Effect of Experimental Variables ............................186

3. 1. Effect of Temperature .................................186

3. 2. Effect of Magnetic Field .................................188

4. Spin-Spin Equilibrium ...................................189

Appendix $A$

Nomenclature of Mössbauer Spectroscopy.............................191 Appendix $B$

Bibliographic Sources..................................................193

Appendix $C$

Selected References on Mössbauer Spectroscopy .........................195

Index 